\title{
Natural selection of academic papers
}

\author{
Pandelis Perakakis • Michael Taylor • \\ Marco Mazza • Varvara Trachana
}

Received: 16 December 2009/Published online: 22 June 2010

(C) Akadémiai Kiadó, Budapest, Hungary 2010

\begin{abstract}
Academic papers, like genes, code for ideas or technological innovations that structure and transform the scientific organism and consequently the society at large. Genes are subject to the process of natural selection which ensures that only the fittest survive and contribute to the phenotype of the organism. The process of selection of academic papers, however, is far from natural. Commercial for-profit publishing houses have taken control over the evaluation and access to scientific information with serious consequences for the dissemination and advancement of knowledge. Academic authors and librarians are reacting by developing an alternative publishing system based on free-access journals and self-archiving in institutional repositories and global disciplinary libraries. Despite the emergence of such trends, the journal monopoly, rather than the scientific community, is still in control of selecting papers and setting academic standards. Here we propose a dynamical and transparent peer review process, which we believe will accelerate the transition to a fully open and free-for-all science that will allow the natural selection of the fittest ideas.
\end{abstract}

Keywords Academic publishing $\cdot$ Peer review $\cdot$ Ethics

P. Perakakis (ه)

Department of Psychology, University of Granada, Campus Cartuja, 18071 Granada, Spain e-mail: peraka@ugr.es

M. Taylor

Institute for Space Applications and Remote Sensing (ISARS), National Observatory of Athens (NOA), Vas. Pavlou \& I. Metaxa, 15236 Penteli, Greece

M. Mazza

Stranski-Laboratorium für Physikalische und Theoretische Chemie, Technische Universität Berlin, Straße des 17. Juni 135, 10623 Berlin, Germany

V. Trachana

Institute of Biological Research and Biotechnology (IBRB), National Hellenic Research Foundation (NHRF), 48 Vas. Constantinou Ave., 11635 Athens, Greece 


\section{Introduction}

There are currently two dominant factors that determine which articles attract attention, gain recognition, increase their impact and, in general, are selected by the academic community. Firstly, an academic paper is expected to pass a peer review (PR) process of a journal deemed to be prestigious. PR is arranged by an editor and is typically performed by a small number (usually no more than three) anonymous reviewers considered experts on the topic covered by the article. There is great disparity between journals both within and across disciplines. Journal editorial committees can have widely varying standards when it comes to article genre and length, acceptability or rejectability, the expertise of reviewers as well as the length of time allocated to the PR process. Such editorial decisions, combined with a journal's bibliometric ranking, determine the prestige of a journal and have a direct consequence on its measured impact expressed through citations. Although, many meta-data services calculate journal citation indices, the "official" quantitative index globally recognized and taken into account by evaluation committees deciding on tenure, promotions and research grants, is the journal impact factor (IF) calculated by Thomson Reuters. Every year, the IF is estimated for a core of more than 7,500 journals that are considered the "world's most cited, peer-reviewed journals" (Thomson. Journal Citation Reports). This system has resulted in the emergence of a journal monopoly, where a small core of high IF journals stands out above a sea of less visible, influential or cited journals. This journal monopoly is also partly responsible for the dramatic increase in individual and bundled journal subscription rates during the past four decades (Van Orsdel and Born 2008).

The "toll for access" required by publishers is the other parameter that affects an article's visibility and impact since elevated subscription costs force libraries to cancel subscriptions to least used or least cost-effective journals (Bergstrom and Bergstrom 2004). This model discriminates even more strongly against developing countries who have lower library budgets (Chan et al. 2006). It is clear that journal controlled PR and overpriced subscriptions have created and sustain a publication system characterised by slow and restricted access to scholarly information as well as an un-natural selection of academic ideas (Taylor et al. 2008).

However, the electronic medium and the World Wide Web now offer the academic community the means to radically modify the way scientific knowledge is communicated, navigated and evaluated by scholars. The Open Access (OA) movement has made significant steps in this direction and has already demonstrated its potential. The Directory of Open Access Journals (DOAJ) currently lists 4197 journals from which 1538 are searchable at article level (DOAJ 2009). In addition, a great number of "pay-for-access" journals allow parallel self-archiving by authors in institutional and/or global discipline archives. In a recent survey of more than 10,000 journals, 90\% were found to allow self-archiving (Romeostats 2009). In spite of these major advances, commercial publishers still control the PR process, preserving their monopolies and justifying high subscription rates and restricted access.

Below we present an alternative model which can open the way to natural selection of academic papers (NSAP) in the academic community (Fig. 1).

\section{Self-archiving}

The first and necessary step for NSAP is mandated self-archiving of all scholarly material to disciplinary or institutional online repositories (IORs). Self-archiving is common 


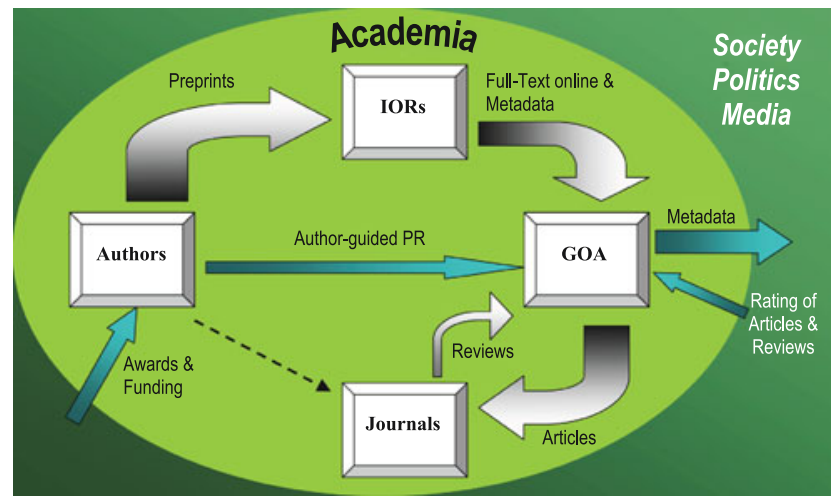

Fig. 1 A graphic representation of the NSAP model. Authors submit their manuscripts to Institutional Online Repositories (IORs). The full-text versions of the manuscripts are indexed in a Global Open Archive (GOA). Authors initiate the process of "author-guided peer review" inviting other academics to openly evaluate their manuscripts available at the GOA. Journals specializing in specific areas select and publish articles that receive positive evaluations. Journals also invite distinguished researchers to write reviews for topics of interest, and continue publishing correspondence, letters etc. The dotted line connecting authors and journals indicates that in the first steps towards the NSAP model authors can continue submitting their manuscripts to academic journals, and at the same time make them available at the corresponding IORs

practice in disciplines like physics and computer science and is now spreading rapidly to other fields. The Registry of Open Access Repositories (ROAR) reports 1351 online repositories and the number keeps rising (ROAR 2009). Although still many scientists appear reluctant to self-archive their articles if they are not required to do so, official mandates by universities and governmental funding agencies are expected to increase selfarchiving rates (Harnad et al. 2008).

\section{Global open archive}

The second step towards NSAP involves the development of a Global Open Archive (GOA) where citation information and links to full-text versions of all papers published at IORs will be made electronically available. There are already important global archives harvesting meta-data and bibliometric information from a large number of IORs using the open access initiative's protocol (OAI-PHM). Once the data is available at the global archive, open access tools such as CiteBase and CiteSeer, can then be used to assess bibliometric information, such as citation indices, collaboration networks and download hits to build up the meta-data records linked to each full-text article.

\section{Author-guided peer review}

Full text articles will at first appear on the GOA with tags to authors' names and affiliations to their host institute or research centers. Initially articles will go online without any quality indicators. Subsequently, the author of the article will be able to personally invite scholars to review and evaluate their manuscript. An unlimited number of invitations can be sent to academics including experts whose opinion is highly esteemed among the academic community. The authors and scholars that agree to review a paper will have to post the 
complete review (comments, corrections and recommendations) on the GOA so that they are readily accessible and linked to the original paper. In addition they will also have to quantitatively evaluate the paper based on pre-established multi-parameter criteria that could be area-specific (Buela-Casal et al. 2006). In this way the whole review process will become absolutely transparent as reviewers sign the review personally. A further iteration would include readers rating both the review and the peer-reviewer creating natural selection also in the PR process. Reviewers will then be rewarded for thorough and scientifically justified reviews and can build up their own academic profiles also linked through the GOA.

Through this transparent peer-review process the authors are able to gradually build the prestige and qualitative assessment of their articles. When a funding agency, a university committee or interested reader performs a search on the GOA, in addition to the general information relating to articles they retrieve (authors, affiliation, abstract, citations etc), they will also be informed of the number of reviews that each article has received, as well as a qualitative evaluation. Readers can then, better than ever, make an assessment about the quality of the paper and its actual or potential impact on the academic community. Furthermore, authors will be able to continually expand and improve their articles based on the comments of invited reviewers and other scholars who will also be able to make signed comments on the GOA. In this way articles become the "seed" of a continuously evolving dynamical process. Authors will have the liberty to decide when to proceed to a new publication either putting forward an entirely new idea, or after what they may consider a significant "update" to previously published research. In this evaluation system the number of articles is no longer as important as the number of reviews, comments, citations or downloads to the new "dynamical articles". This will reduce the number of articles that today are being published more for their inflationary potential regarding impact factors rather than for novel academic merit, and eliminate the modern trend of recycling old ideas already published in a different journal. It will also be easier to track the research history of a single author or research group as this information will be more concentrated in a smaller number of articles. At the same time, the GOA could follow the lead of preprint servers such as arXiv or NASA-ADS where links to prior versions are preserved in order to maintain the chronological development of ideas and the consistency of references.

This proposed mechanism will accelerate the open and transparent exchange of ideas that today occurs in any formal or informal academic conference, but which is absent from the present PR process. Articles submitted to IORs will be judged by a substantially greater number of experts. Furthermore, ideas proposed in a manuscript will be subject to constant scrutiny over time with the fittest or most prolific innovations being selected in much the same way as genes or "memes" (Dawkins 2006) are selected. Ideas will be fully immersed in their proper environment, the academic community, and judged by merit weeding out conflicts of interest. In addition, the ranking of review ratings could be very easily implemented as a way of filtering the most insightful comments (this strategy has proved a key factor in boosting the quality assessment of commercial products such as Amazon.com who made $\$ 2.7$ billion using such a method).

\section{Implications of the NSAP: a glimpse into the future of academic publishing}

In this section we briefly examine the immediate implications of the NSAP model for academic journals, universities and institutions, librarians and bibliometricians and academic authors themselves. 
Journals

One of the first questions that arises from our suggested model is: what will happen to traditional academic journals? The answer is that journals need not perish but rather radically transform their function. Journals in the NSAP model will be able to adopt a new and more important role: that of selecting the most highly evaluated articles from the vast pool of information provided by subject-based repositories and global archives. They will continue to invite authors to submit reviews of interesting or controversial topics, and provide general and important insights through newsletters, commentaries and editorials. They will no doubt charge for these valuable services, but these fees will now not be able to place financial or electronic access barriers on academic research.

\section{Institutes and universities}

The first consequence of the NSAP model for universities and institutes is the significant reduction in the costs spent annually on subscriptions to journals. Although there will still be a need for journal subscriptions, the prices will drastically drop since access to scholarly information will be freely available through the GOA. A part of the income from reduced subscription prices can be used for organizing and maintaining IORs at no extra cost to institutes and research centers. Each university will also now be responsible for the papers they edit, format and publish at their repositories. This will be one way that universities may use NSAP to build their own prestige, as they will have the freedom to establish and apply their own criteria for the acceptance of papers to be published at their IOR. As exemplified by the Massachusetts Institute of Technology (MIT), a university could decide to submit each paper originating from its faculty to a PR process in order to be accepted for publication on its IOR. Thus, a paper on the GOA originating from MIT will have an additional quality assurance prior to public review.

\section{Librarians and bibliometricians}

The application of the NSAP publication model has important implications for librarians and the science of bibliometrics in general. Bibliometrics as a science will inevitably evolve as the GOA will provide a lot more information on papers than simple citation indices. Important new data such as statistics of visits and downloads from article pages will offer new insights on international academic collaboration patterns. New indices and network statistics will be developed with the addition of reviewer information previously inaccessible. One can speculate on the development also of multivariate quality indices.

\section{Authors}

Academic authors will primarily benefit from the NSAP model. In-press publication time will be significantly reduced or eliminated altogether. From the moment the paper is accepted by an institute's evaluation committee, it is already publicly available through the IOR and subsequently, the GOA. Researchers all over the world will have unrestricted, full-text access to the totality of academic output without having to be affiliated to a university or research center and without having to pay subscriptions or download charges to profit-oriented publishing houses. At the same time, an unaffiliated researcher will be equally able to submit a paper to the GOA and invite reviewers in the same way as affiliated authors. This will widen the pool of innovative ideas. Perhaps, an independent 
international institution can be developed whose role will be to organize a special repository for papers originating from unaffiliated authors so as to guarantee a quality minimum prior to publication at GOA. After publishing at the GOA, authors are free to manage the fate of their own work. Achieving a positive review by a major expert in the field would be synonymous with what is considered today to publish in a high impact factor journal. However, publishing in a prestigious journal does not inform the reader of the quality of the PR a paper has received. Readers' perceptions of quality are subconsciously pre-selected by the IF of the journal. On the contrary, NSAP guarantees transparency and more objective quality assessment.

Every field of academia has evolved towards high specialization. It is therefore common for a reviewer to have to judge the manuscript of a colleague or competitor. This situation often promotes incestuous or unethical conduct. One of the principal causes is the small number of anonymous reviewers involved. A transition to a system of large-scale and transparent PR would certainly improve this issue because conflicts of interest would be selected against or be diluted in the averaging that will take place over the multiple opinions. Clearly, traditional journals can do very little to address this issue. Furthermore, NSAP will also help to solve the problem of academic "families" that have come to monopolize entire disciplines. In a transparent system, the careers and reputation of the reviewers is also at stake and will bring a halt to biased support for academic papers of questionable quality.

We believe the proposed model is self-sustaining, ecological and robust to economic fluctuations. Every party to the publishing process stands to gain. An accomplished scientist participating as a reviewer will be exposed to a variety of novel ideas stimulating his or her own investigations. And, at the other end of the spectrum, a young scientist would gain a respectable position in his or her field by providing insightful comments or by highlighting connections to other published work. As a case in point, a young lover of mathematics in an isolated rural village with an internet connection will, not only have unlimited access to academic papers, but also be able to publish his or her own ideas and submit them to the process of transparent peer-review, healthy competition and natural selection based on quality and academic relevance. If these ideas are good then scholars that will be the first to recognize this and actively participate in the dissemination of the paper will also raise their own academic status.

\section{Conclusion}

In this brief manuscript we present a novel publication model, the NSAP, that addresses the fundamental problems of dissemination of knowledge, the deficiencies of the PR process as well as issues associated with journal monopolies. We believe that, although the details of this model are likely to evolve and be refined during implementation, NSAP or some equivalent model will eventually become a reality, and an inevitable one driven by and dictated by the needs of the academic community and the society in general. The internet and the remarkable advancement of free publishing software have created the preconditions to make this possible. Here, we have identified three necessary steps and we have seen that the first two are already becoming a reality.

One of the main advantages of the NSAP model is that it can be developed in parallel with the current publication system until it eventually replaces it and without the necessity for abrupt changes. Following the example of MIT (Plotkin 2009), universities and research institutes can start establishing their publication criteria and organise their IORs. 
At the same time a new or an existing meta-database protocols like the OAI-PMH can harvest these institutional digital libraries and develop data-mining and bibliometric analysis tools in the spirit of CiteBase and CiteSeer. When the global database we called GOA is launched and functional, authors can start inviting scholars to review and evaluate their papers. This procedure can start taking place in parallel to submission to traditional journals transforming the nature of copyright which will remain firmly in the hands of authors.

This is a special time in the history of academic publishing. The NSAP model stands on the shoulders of initiatives already evolving and it appears that we shall see in the near future a gradual and radical transformation of the academic publishing landscape towards a process of natural selection of scientific ideas.

\section{References}

Bergstrom, C. T., \& Bergstrom, T. C. (2004). The costs and benefits of library site licenses to academic journals. Proceedings of the National Academy of Sciences, 101(3):897-902.

Buela-Casal, G., Perakakis, P., Taylor, M., \& Checa, P. (2006). Measuring internationality: Reflections and perspectives on academic journals. Scientometrics, 67(1):45-65.

Chan, L., Kirsop, B., \& Arunachalam, S. (2006). Open access archiving: The fast track to building research capacity in developing countries. Science and Development Network, 1.

Dawkins, R. (2006). The selfish gene. NY, USA: Oxford University Press.

Directory of open access journals (DOAJ). (2009). http://www.doaj.org. Accessed 3 June.

Harnad, S., Brody, T., Vallières, F., Carr, L., Hitchcock, S., Gingras, Y. et al. (2008). The access/impact problem and the green and gold roads to open access: An update. Serials Review , 34(1):36-40.

Plotkin, N. (2009). MIT will publish all faculty articles free in online repository. http://tech.mit.edu/V129/ N14/open\_access.html. Accessed 4 July.

Registry of Open Access Repositories (ROAR). (2009). http://roar.eprints.org/. Accessed 3 June.

Romeostats. (2009). Journal policies—summary statistics so far. http://romeo.eprints.org/stats.php. Accessed 3 June.

Taylor, M., Perakakis, P., \& Trachana, V. (2008). The siege of science. Ethics in Science and Environmental Politics (ESEP), 8(1), 17-40.

Thomson. Journal Citation Reports. http://thomsonreuters.com/products_services/scientific/Journal_ Citation $\_$Reports. Accessed 3 June 2009.

Van Orsdel, L. C., \& Born, K. (2008). Periodicals price survey 2008 embracing openness. Library Journal, 133(7), 6. 